\title{
A COMPENSATED FUZZY HOPFIELD NEURAL NETWORK FOR CODEBOOK DESIGN IN VECTOR QUANTIZATION
}

\author{
SHAO-HAN LIU and JZAU-SHENG LIN* \\ Department of Electronic Engineering \\ National Chin-Yi Institute of Technology \\ No. 35, Lane 215, Sec. 1, Chun Shan Road \\ Taichung, Taiwan, R.O.C. \\ *E-mail: jslin@chinyi.ncit.edu.tw
}

\begin{abstract}
In this paper, a new Hopfield-model net called Compensated Fuzzy Hopfield Neural Network (CFHNN) is proposed for vector quantization in image compression. In CFHNN, the compensated fuzzy c-means algorithm, modified from penalized fuzzy cmeans, is embedded into Hopfield neural network so that the parallel implementation for codebook design is feasible. The vector quantization can be cast as an optimal problem that may also be regarded as a minimization of a criterion defined as a function of the average distortion between training vector and codevector. The CFHNN is trained to classify the divided vectors on a real image into feasible class to generate an available codebook when the defined energy function converges to near global minimum. The training vectors on a divided image are mapped to a two-dimensional Hopfield neural network. Also the compensated fuzzy c-means technique is used to update the quantization performance and to eliminate searching for the weighting factors. In the context of vector quantization, each training vector on the divided image is represented by a neuron which is fully connected by the other neurons. After a number of iterations, neuron states are refined to reach near optimal result when the defined energy function is converged.
\end{abstract}

Keywords: Hopfield neural network; fuzzy c-means; penalized fuzzy c-means; vector quantization.

\section{INTRODUCTION}

Robust identification for image processing needs data compression that preserves the features in the original image. In image compression, the process of codebook design from training vectors of divided images is a very important step in vector quantization. A great deal of literature based on vector quantization has been discussed in other articles. ${ }^{2,4,9,10,14,17-19,24,28}$ The goal of vector quantization is to create a codebook for which the average distortion generated by a training vector and a codevector in codebook is minimized. The minimization of average distortion is widely used by a gradient descent-based iterative procedure. According to the cluster centroid in the previous iteration and nearest neighbor rule, a positive improvement to update the codebook is performed iteratively.

Image compression is the coding of transformed image using a code of fixed or variable length. Vector quantization is a significant methodology in image compression, in which blocks of divided pixels are formed as training vectors rather than

*Author for correspondence. 
individual scales. Such a method results in the massive reduction of the image information in image transmission. The image is reconstructed by replacing each image block with its nearest codevector. The dimensions, with $N \times N$ pixels in an image, can be divided into $n$ blocks (vectors of pixels) and each block occupies $\lambda \times \lambda(\lambda<N)$ pixels. A vector quantization is a technique that maps training vectors $\left\{\mathbf{X}_{x}, x=1,2, \ldots, n\right\}$ in Euclidean $\lambda \times \lambda$-dimensional space $\mathrm{R}^{\lambda \times \lambda}$ into a set of $\left\{\mathbf{Y}_{x}, x=1,2, \ldots, n\right\}$ points in $\mathrm{R}^{\lambda \times \lambda}$, called a codebook. The mapping is usually defined to minimize expected distortion measure, $E\left[d\left(\mathbf{X}_{x}, \mathbf{Y}_{x}\right)\right]$, using the mean square error (MSE) given by $d(\mathbf{x}, \mathbf{y})=(\mathbf{x}-\mathbf{y})^{T}(\mathbf{x}-\mathbf{y})$.

The codebook design is the primary problem in image compression based on vector quantization. Codebook design can be considered as a clustering process in which the training vectors are classified into the specific classes based on the minimization of average distortion between the training vectors and codebook vectors (classes' centers). Then the clustering algorithms perform a positive improvement to update the codebook iteratively. In addition to the conventional technique, the neural network technique has also been demonstrated to address codebook design problems. In this article, a compensated Fuzzy Hopfield Neural Network (CFHNN) is proposed for codebook design in vector quantization. In CFHNN, the problem of the vector quantization is regarded as a process of the minimization of a cost function. This cost function is defined as the average distortion between the training vectors in a divided image to the cluster centers represented by the codevectors in the codebook. The structure of this network is constructed as a two-dimensional fully interconnected array with the columns representing the number of codevectors (clusters) and the rows representing the training vectors in the divided image. However, a training vector does not necessarily belong to one class. Instead, a certain membership grade belonging to the proper class is associated with every vector sample. In addition to the fuzzy reasoning strategy, a compensated term is added as input bias to update the performance of the training process. In CFHNN, an original Hopfield network is modified and the compensated fuzzy c-means strategy is added. Consequently, the energy function can be quickly converged into a near global minimum in order to produce a satisfactory codebook. Compared with conventional techniques, the major strength of the presented CFHNN is computationally more efficient due to the inherent parallel structures and valuable compensated fuzzy c-means strategy. In a simulated study, the CFHNN is described to have the capability for vector quantization in image compression and show promising results.

The remainder of this paper is organized as follows. Section 2 discusses fuzzy cmeans, penalized fuzzy c-means and compensated fuzzy c-means techniques; Sec. 3, proposes the vector quantization by the CFHNN; Sec. 4 presents several experimental results; Sec. 5 gives the discussion and conclusions; and finally the convergence of the CFHNN is shown in Appendix. 


\section{FUZZY C-MEANS, PENALIZED FUZZY C-MEANS AND COMPENSATED FUZZY C-MEANS}

The fuzzy set theory since introduced by Zadeh ${ }^{27}$ in 1965 has been applied in various fields. The theory of fuzzy logic provides a mathematical environment to capture the uncertainties much the same as human cognition processes. The fuzzy c-means (FCM) clustering strategy was first described by Dunn, ${ }^{7}$ and an associated conception and strategy was proposed by Bezdek. ${ }^{3}$ The fuzzy clusters are generated by the partition of training samples in accordance with the membership functions matrix $U=\left[\mu_{x, i}\right]$. The component $\mu_{x, i}$ denotes the degree of possibility that a training sample $z_{x}$ belongs to an $i$ th fuzzy cluster. The objective function of FCM is defined as

$$
J_{\mathrm{FCM}}=\frac{1}{2} \sum_{x=1}^{n} \sum_{i=1}^{c}\left(\mu_{x, i}\right)^{m}\left\|z_{x}-\varpi_{i}\right\|^{2}
$$

where $|\cdot|$ is the Euclidean distance between the training sample and cluster center.

The penalized fuzzy c-means algorithm (PFCM), a penalty term embedded into FCM, was demonstrated by Yang. ${ }^{25,26}$ It is a class of fuzzy classification extended with the addition of a penalty term and based on the maximum likelihood procedure. PFCM was demonstrated to be more meaningful and effective than FCM by Yang. ${ }^{25}$ For the clustering problem, the PFCM energy function is defined as follows:

$$
\begin{aligned}
J_{\mathrm{PFCM}} & =\frac{1}{2} \sum_{x=1}^{n} \sum_{i=1}^{c} \mu_{x, i}^{m}\left\|z_{x}-\varpi_{i}\right\|^{2}-\frac{1}{2} \nu \sum_{x=1}^{n} \sum_{i=1}^{c} \mu_{x, i}^{m} \ln \alpha_{i} \\
& =J_{\mathrm{FCM}}-\frac{1}{2} \nu \sum_{x=1}^{n} \sum_{i=1}^{c} \mu_{x, i}^{m} \ln \alpha_{i}
\end{aligned}
$$

where $\alpha_{i}$ is a proportional constant of class $i$ and $\nu \geq 0$ is also a constant. When $\nu=0, J_{\mathrm{PFCM}}$ equals the energy function of fuzzy c-means algorithm. The penalty term, $-\frac{1}{2} \nu \sum_{x=1}^{n} \sum_{i=1}^{c} \mu_{x, i}^{m} \ln \alpha_{i}$ is added to the objective function and $\alpha_{i}, \varpi_{i}$ and $\mu_{x, i}$ are redefined as

$$
\begin{aligned}
\alpha_{i} & =\frac{\sum_{x=1}^{n} \mu_{x, i}^{m}}{\sum_{x=1}^{n} \sum_{i=1}^{c} \mu_{x, i}^{m}} ; \quad i=1,2, \ldots c \\
\varpi_{i} & =\frac{\sum_{x=1}^{n} \mu_{x, i}^{m} z_{x, i}}{\sum_{x=1}^{n} \mu_{x, i}^{m}}
\end{aligned}
$$

and

$$
\begin{gathered}
\mu_{x, i}=\left(\sum_{\ell=1}^{c} \frac{\left(\left\|z_{x}-\varpi_{i}\right\|^{2}-\nu \ln \alpha_{i}\right)^{1 /(m-1)}}{\left(\left\|z_{x}-\varpi_{\ell}\right\|^{2}-\nu \ln \alpha_{\ell}\right)^{1 /(m-1)}}\right)^{-1} ; \\
x=1,2, \ldots, n ; i=1,2 \ldots, c
\end{gathered}
$$


The PFCM algorithm is presented for the clustering problem as follows:

\section{PFCM algorithm}

Step 1: Randomly initialize class centers $\varpi_{i}(2 \leq i \leq c)$ and fuzzy c-partition $U^{(0)}$. Give fuzzification parameter $m(1 \leq m<\infty)$, constant $\nu$, and the value $\varepsilon>0$.

Step 2: Compute $\alpha_{i}^{(t)}, \varpi_{i}^{(t)}$ with $U^{(t-1)}$ using Eqs. (3) and (4). Calculate the membership matrix $U=\left[\mu_{x, j}\right]$ with $\alpha_{i}^{(t)}, \varpi_{i}^{(t)}$ using Eq. (5).

Step 3: Compute $\Delta=\max \left(\left|U^{(t+1)}-U^{(t)}\right|\right)$. If $\Delta>\varepsilon$, then go to Step 2; otherwise go to Step 4.

Step 4: Find the results for the final class centers.

Yang has proved the convergence of $J_{\mathrm{PFCM}}$ in Ref. 25. But the penalty degree is too heavy to rapidly converge. The penalty term $-\frac{1}{2} \nu \sum_{x=1}^{n} \sum_{i=1}^{c} \mu_{x, i}^{m} \ln \alpha_{i}$ in PFCM is replaced by a compensated term $+\frac{1}{2} \nu \sum_{x=1}^{n} \sum_{i=1}^{c} \mu_{x, i}^{m} \tanh \left(\alpha_{i}\right)$ then the energy function and membership function in a so-called Compensated Fuzzy CMeans (CFCM) algorithm is defined as

$$
\begin{aligned}
J_{\mathrm{CFCM}} & =\frac{1}{2} \sum_{x=1}^{n} \sum_{i=1}^{c} \mu_{x, i}^{m}\left\|z_{x}-\varpi_{i}\right\|^{2}+\frac{1}{2} \nu \sum_{x=1}^{n} \sum_{i=1}^{c} \mu_{x, i}^{m} \tanh (\alpha) \\
& =J_{\mathrm{FCM}}+\frac{1}{2} \nu \sum_{x=1}^{n} \sum_{i=1}^{c} \mu_{x, i}^{m} \tanh \left(\alpha_{i}\right)
\end{aligned}
$$

and

$$
\begin{gathered}
\mu_{x, i}=\left(\sum_{\ell=1}^{c} \frac{\left(\left\|z_{x}-\varpi_{i}\right\|^{2}+\nu \tanh \left(\alpha_{i}\right)\right)^{1 /(m-1)}}{\left(\left\|z_{x}-\varpi_{\ell}\right\|^{2}+\nu \tanh \left(\alpha_{\ell}\right)\right)^{1 /(m-1)}}\right)^{-1} \\
x=1,2, \ldots, n ; i=1,2 \ldots, c
\end{gathered}
$$

where $\alpha_{i}$ and $\nu$ are the same definition as Eq. (5). Equation (6) can be rewritten as

$$
\begin{aligned}
J_{\mathrm{CFCM}} & =J_{\mathrm{FCM}}+\frac{1}{2} \nu \sum_{x=1}^{n} \sum_{i=1}^{c} \mu_{x, i}^{m} \tanh \left(\alpha_{i}\right) \\
& =J_{\mathrm{FCM}}-\frac{1}{2} \nu \sum_{x=1}^{n} \sum_{i=1}^{c} \mu_{x, i}^{m} \tanh \left(-\alpha_{i}\right) .
\end{aligned}
$$

Since $0<\alpha_{i}<1$, we can find $\tanh \left(-\alpha_{i}\right) \subset \ln \left(\alpha_{i}\right)$ which implies that $J_{\mathrm{CFCM}}$ can also be convergent.

\section{COMPENSATED FUZZY HOPFIELD NETWORK FOR VECTOR QUANTIZATION}

The Hopfield neural network, with simple architecture and parallel potential, has been applied in many fields. ${ }^{1,5,6,11,12,15,16,21-23}$ Chung et al. ${ }^{6}$ used the discrete 
Hopfield neural network with competitive learning (called CHNN) to polygonal approximation. In Ref. 6, the winner-take-all scheme has been adopted in the two-dimensional discrete Hopfield neural network to eliminate the need for finding weighting factors in the energy function. Amatur et al. ${ }^{1}$ applied the continuous Hopfield neural network with competitive learning for the segmentation of multispectral MR brain images. Endocardial boundary detection using the Hopfield neural network was described by Tsai et al. ${ }^{22}$; Washizawa ${ }^{23}$ applied the Hopfield neural network to emulate saccades; optimal guidance using the Hopfield neural network was presented by Steck et al. ${ }^{21}$ Lin et al. ${ }^{15,16}$ proposed fuzzy Hopfield neural network (called FHNN) to medical image segmentation. In the conventional Hopfield network or CHNN, a neuron $(x, i)$ in a firing state indicates that sample $z_{x}$ belongs to class $i$. But, in the FHNN, a neuron $(x, i)$ in a fuzzy state indicates that sample $z_{x}$ belongs to class $i$ with a degree of uncertainty described by a membership function. Following the same ideal of FHNN, the author also used the Hopfield neural network with compensated fuzzy c-means strategy (CFHNN) for vector quantization in image compression. In this paper, the two-dimensional neuron array is used. Each row is assigned to a training vector ( $n$ rows) with $\ell \times \ell$ pixels and each column is regarded as a cluster center (codevector) in the CFHNN. If $c$ is the number of codevectors being prechosen, then the CFHNN consists of $n \times c$ neurons which can be conceived as a two-dimensional array which occupies $n$ rows and $c$ columns.

The CFHNN uses the Hopfield neural network architecture with CFCM strategy to classify the training vectors in the divided image to generate a feasible codebook. In order to increase the capability of the proposed approach, the energy function is formulated on the basis of within-class scatter matrix, a concept widely used in pattern classification. Here, the within-class scatter matrix is defined by the average distortion between the training vector and codevector within the same class. Let $\mu_{x, i}$ be the fuzzy state of the $(x, i)$ th neuron and $\mathbf{W}_{x, i ; y, i}$ present the interconnected vector weight between neuron $(x, i)$ and neuron $(y, i)$. A neuron $(x, i)$ in the network receives weighted inputs $\mathbf{W}_{x, i ; y, i}$ from each neuron $(y, i)$ and a bias $I_{x, i}$ from output. The total input to neuron $(x, i)$ is computed as

$$
\operatorname{Net}_{x, i}=\left|\mathbf{z}_{x}-\sum_{y=1}^{n} \mathbf{W}_{x, i ; y, i}\left(\mu_{y, i}\right)^{m}\right|^{2}+I_{x, i} .
$$

The modified Lyapunov energy function of the two-dimensional Hopfield neural network using CFCM strategy is given by

$$
E=\frac{1}{2} \sum_{x=1}^{n} \sum_{i=1}^{c}\left(\mu_{x, i}\right)^{m}\left|\mathbf{z}_{x}-\sum_{y=1}^{n} \mathbf{W}_{x, i ; y, i}\left(\mu_{y, i}\right)^{m}\right|^{2}+\frac{1}{2} \sum_{x=1}^{n} \sum_{i=1}^{c} I_{x, i}\left(\mu_{x, i}\right)^{m}
$$

where $|\cdot|$ is the average distortion between the training vector to cluster center on the divided image, $\sum_{y=1}^{n} \mathbf{W}_{x, i ; y, i}$ is the total weighted input received from neuron $(y, i)$ in row $i, \mu_{x, i}$ is the output state at neuron $(x, i)$, and $m$ is the fuzzification parameter. Each column of this modified Hopfield network represents a class and each row represents a training vector. The network reaches a stable state when the 
modified Lyapunov energy function is minimized. For example, a neuron $(x, i)$ in a maximum membership state indicates that training vector $\mathbf{z}_{x}$ belongs to class $i$.

The objective function, used to generate a suitable codebook that has a minimum average distortion between training vector and the cluster centroid within class, is given by

$$
\begin{aligned}
E= & \frac{A}{2} \sum_{x=1}^{n} \sum_{i=1}^{c}\left(\mu_{x, i}\right)^{m}\left|\mathbf{z}_{x}-\sum_{y=1}^{n} \frac{1}{\sum_{h=1}^{n}\left(\mu_{h, i}\right)^{m}} \mathbf{z}_{y}\left(\mu_{y, i}\right)^{m}\right|^{2} \\
& +\frac{B}{2}\left[\left(\sum_{x=1}^{n} \sum_{i=1}^{c} \mu_{x, i}\right)-n\right]^{2}+\frac{1}{2} \nu \sum_{x=1}^{n} \sum_{i=1}^{c}\left(\mu_{x, i}\right)^{m} \tanh \left(\alpha_{i}\right)
\end{aligned}
$$

where $E$ is the total intra-class scatter energy that accounts for the scattered energies distributed by all training vectors in the same class with a membership grade, and both $\mathbf{z}_{x}$ and $\mathbf{z}_{y}$ are the training vectors at rows $x$ and $y$ respectively. The proportional constant of class $i, \alpha_{i}$ and constant $\nu$ have the same definition as $J_{\mathrm{CFCM}}$.

The first term in Eq. (11) is the within-class scatter energy that is the average distortion between training vector and the cluster centroid over $c$ clusters. The second term guarantees that the $n$ training vectors in $\mathbf{Z}$ can only be distributed among these $c$ clusters. More specifically, the second term imposes constraints on the objective function and the first term minimizes the intra-class Euclidean distance from training vector to the cluster centroid in any given cluster. The last term is the compensated term same as for the definition in Eq. (6) of CFCM algorithm.

As mentioned in Ref. 6, the quality of classification result is very sensitive to the weighting factors. Searching for optimal values of these weighting factors is expected to be tedious and time-consuming. To alleviate this problem, a 2-D Hopfield neural network with compensated fuzzy c-means clustering strategy, called CFHNN is proposed so that the constrain terms can be handled more efficiently. All the neurons on the same row compete against one another to determine which neuron is the maximum membership value belonging to class $i$. In other words, the summation of the membership grade of states in the same row equals 1 , and the total membership states in all $n$ rows equal $n$. It is also ensured that all training vectors will be classified into these $c$ classes. The modified Hopfield neural network CFHNN enables the scatter energy function to converge rapidly into a minimum value. Then, the scatter energy of the CFHNN can be further simplified as

$$
\begin{aligned}
E= & \frac{1}{2} \sum_{x=1}^{n} \sum_{i=1}^{c}\left(\mu_{x, i}\right)^{m}\left|\mathbf{z}_{x}-\sum_{y=1}^{n} \frac{1}{\sum_{h=1}^{n}\left(\mu_{h, i}\right)^{m}} \mathbf{z}_{y}\left(\mu_{y, i}\right)^{m}\right|^{2} \\
& +\frac{1}{2} \nu \sum_{x=1}^{n} \sum_{i=1}^{c}\left(\mu_{x, i}\right)^{m} \tanh \left(\alpha_{i}\right) .
\end{aligned}
$$

By using Eq. (12), which is a modification of Eq. (11), the minimization of energy $E$ is greatly simplified since it contains only two terms and hence the requirement of having to determine the weighting factors A and B vanishes. Comparing Eq. (12) 
with the modified Lyapunov function Eq. (10), the synaptic interconnection weights and the bias input can be obtained as

$$
\mathbf{W}_{x, i ; y, i}=\frac{1}{\sum_{h=1}^{n}\left(\mu_{h, i}\right)^{m}} \mathbf{z}_{y}
$$

and input bias

$$
I_{x, i}=\nu \tanh \left(\alpha_{i}\right) .
$$

By introducing Eqs. (13) and (14) into Eq. (9), the input to neuron $(x, i)$ can be expressed as

$$
\operatorname{Net}_{x, i}=\left|\mathbf{z}_{x}-\sum_{y=1}^{n} \frac{1}{\sum_{h=1}^{n}\left(\mu_{h, i}\right)^{m}} \mathbf{z}_{y}\left(\mu_{y, i}\right)^{m}\right|^{2}+\nu \tanh \left(\alpha_{i}\right) .
$$

Consequently, the state (i.e. membership function) for the neuron $(x, i)$ is given as

$$
\mu_{x, i}=\left[\sum_{j=1}^{c}\left(\frac{\operatorname{Net}_{x, i}}{\operatorname{Net}_{x, j}}\right)^{1 / m-1}\right]^{-1} \quad \text { for all } i .
$$

Using Eqs. (13), (15) and (16), the CFHNN can classify $c$ clusters in a parallel manner that is described as follows:

\section{CFHNN Algorithm}

Step 1: Input a set of training vector $\mathbf{Z}=\left\{\mathbf{z}_{1}, \mathbf{z}_{2}, \ldots, \mathbf{z}_{n}\right\}$, fuzzification parameter $m(1 \leq m<\infty)$, the number of clusters $c$, constant $\nu$, and initialize the states for all neurons $U=\left[\mu_{x, i}\right]$ (membership matrix).

Step 2: Compute $\alpha_{i}$ and weighted matrix using Eqs. (3) and (13) respectively.

Step 3: Calculate the input to each neuron $(x, i)$ :

$$
\operatorname{Net}_{x, i}=\left|\mathbf{z}_{x}-\sum_{y=1}^{n} \frac{1}{\sum_{h=1}^{n}\left(\mu_{h, i}\right)^{m}} \mathbf{z}_{y}\left(\mu_{y, i}\right)^{m}\right|^{2}+\nu \tanh \left(\alpha_{i}\right) .
$$

Step 4: Apply Eq. (16) to update the neurons' membership values in a synchronous manner:

$$
\mu_{x, i}=\left[\sum_{j=1}^{c}\left(\frac{\operatorname{Net}_{x, i}}{\operatorname{Net}_{x, j}}\right)^{1 / m-1}\right]^{-1} \quad \text { for all } i
$$

Step 5: Compute $\Delta=\max \left(\left|U^{(t+1)}-U^{(t)}\right|\right)$. If $\Delta>\varepsilon$, then go to Step 2, otherwise go to Step 6.

Step 6: Find the codebook for the final membership matrix.

In Step 3, the inputs are calculated for all neurons. In Step 4, the compensated fuzzy c-means clustering method is applied to determine the fuzzy state with the synchronous process. Here, a synchronous iteration is defined as an updated fuzzy state for all neurons. 


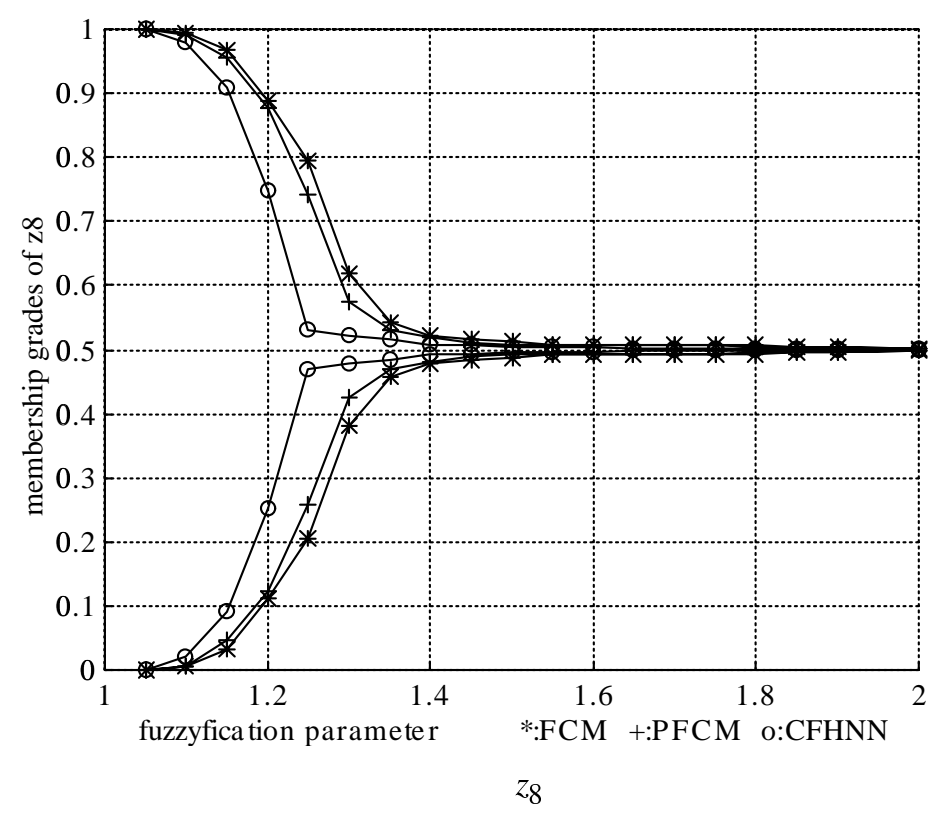

Fig. 1. The membership grade curve of pattern $z_{8}$ with $c=2$ in different algorithms.

To see the performance of the FCM, PFCM, and the proposed algorithm CFHNN, the Butterfly example given by Ruspini ${ }^{20}$ and $\mathrm{Jou}^{13}$ for fuzzy clustering problem is considered. Patterns 7, 8 and 9 construct a bridge between the wings of the butterfly with 15 input patterns in $R^{2}$. The membership grades are nearly symmetric with respect to pattern $z_{8}$ in both data coordinate directions for all algorithms. The CFHNN can rapidly result in a symmetric manner for the membership grade of pattern $z_{8}$ with respect to both clusters. This fact can be shown in Fig. 1, where the curves above membership grade $=0.5$ indicate the membership grades of pattern $z_{8}$ belonging to cluster 1 while the curves under membership grade $=0.5$ denote the membership grades of pattern $z_{8}$ belonging to cluster 2 with distinct $m$ from 1.05 to 2.0 for all strategies in this example. In accordance with Fig. 1, the symmetric manner of membership grades can be rapidly reached for the critical patterns in partition clusters using CFHNN.

\section{EXPERIMENTAL RESULTS}

In this paper, the quality of the image reconstructed from the designed codebooks is compared using GLA, FCM, PFCM and CFHNN algorithms, respectively in an IBM compatible Pentium computer. The training vectors were extracted from $256 \times 256$ real images with 8 -bit gray levels, which were divided into $4 \times 4$ blocks to generate 4096 nonoverlapping 16-dimensional vectors. Three codebooks of size 64 , 128 and 256 were generated using these training vectors and the compression rates were 0.5 bits per pixel (bpp), $0.4375 \mathrm{bpp}$ and $0.375 \mathrm{bpp}$, respectively. The peak signal to noise ratio (PSNR) was evaluated in the reconstructed images. Row 1 in 

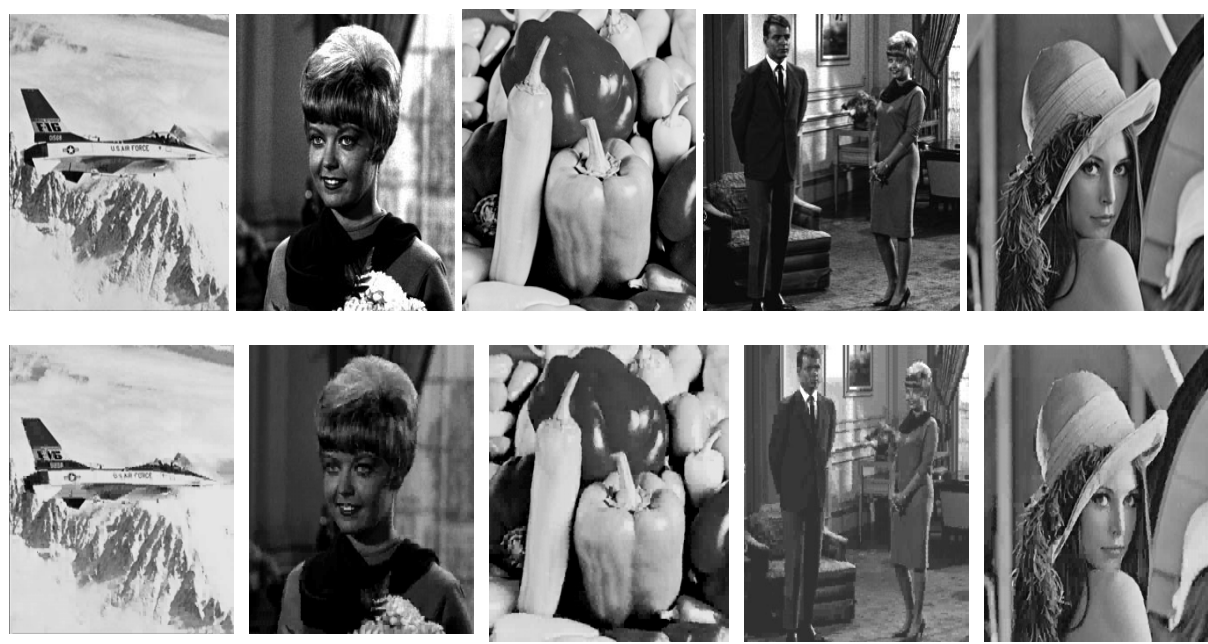

Fig. 2. Original images and reconstructed images with compression ratio $=0.500$ bpp using the CCHNN algorithm: (row 1) are original images; (row 2) are reconstructed images with codebook of size $k=256$.

Table 1. PSNR of images reconstructed from codebook of various sizes by CFHNN with fuzzification parameters $m=1.2$ and $m=1.4$, respectively.

\begin{tabular}{lcccccc}
\hline Codebook size & \multicolumn{2}{c}{64} & \multicolumn{2}{c}{128} & \multicolumn{2}{c}{256} \\
\hline$m$ & 1.2 & 1.4 & 1.2 & 1.4 & 1.2 & 1.4 \\
F16 & 25.860 & 25.012 & 26.989 & 25.877 & 28.465 & 26.621 \\
Baboon & 23.321 & 21.928 & 24.029 & 22.330 & 24.758 & 22.419 \\
Girl & 29.672 & 28.792 & 30.694 & 29.681 & 31.805 & 30.270 \\
Pepper & 26.274 & 25.859 & 27.656 & 26.928 & 29.330 & 27.936 \\
Boy_girl & 30.254 & 29.433 & 31.696 & 30.362 & 33.285 & 30.736 \\
Lenna & 27.756 & 25.974 & 28.827 & 26.771 & 29.977 & 27.313 \\
\hline
\end{tabular}

Fig. 2 shows the training images. Row 2 in Fig. 2 shows the images reconstructed from the codebook design by the proposed CFHNN based on within-class scatter energy with $0.5 \mathrm{bpp}$. The average PSNRs for all reconstructed images completed by the CFHNN is higher with $2 \mathrm{~dB}, 0.4 \mathrm{~dB}$ and $0.2 \mathrm{~dB}$ than those done by GLA, FCM and PFCM, respectively. Table 1 shows the PSNR of the images reconstructed from the various codebooks designed using the CFHNN algorithm with various fuzzification parameters. In accordance with Table 1, better results can be obtained with $m=1.2$ in the proposed CFHNN algorithm. In summary, from the experiment results, the proposed algorithm could satisfactorily produce the codebook design while the network convergence is guaranteed.

The problem of determining the optimal values of the weighting factors is avoided in the CFHNN. It is implied that this new approach is more efficient and versatile than GLA, FCM and PFCM for vector quantization in image compression. Since the CFHNN is highly interconnected and occupies parallel abilities; computation time can be largely reduced by way of parallel processing. 


\section{DISCUSSION AND CONCLUSION}

A two-dimensional Hopfiled neural network based on the within-class scatter matrix using the compensated fuzzy reasoning strategy for vector quantization in image compression has been presented in this paper. From the experimental results, the proposed CFHNN algorithm produces reconstructed images more promising than those reconstructed by the GLA, FCM and PFCM algorithms. The network differs from the conventional Hopfield network in that a compensated fuzzy reasoning strategy is imposed for updating the neuron states. In addition, in Sec. 3 was demonstrated that the CFHNN was more meaningful and effective than PFCM and FCM. Floreen et $a l .{ }^{8}$ indicated that to determine the attraction radius of a stable vector in a discrete (binary) Hopfield network is a NP-hard problem. It might hamper the convergence of the discrete Hopfield net to train with complex and large data sets. In the discrete Hopfield network, a neuron $(x, i)$ in a firing state indicates that sample $\mathbf{z}_{x}$ belongs to class $i$. But, in the CFHNN, a neuron $(x, i)$ in a fuzzy state indicates that sample $\mathbf{z}_{x}$ belongs to class $i$ with a degree of uncertainty described by a membership function. The CFHNN, which is also a continuous model with membership function, can overcome the NP-hard problem exhibit in binary Hopfield net.

The energy function used for the CFHNN is called the scatter energy function that is formulated and based on a widely used concept in pattern classification. The fuzzy reasoning method with a compensated term implemented by the CFHNN greatly simplifies the scatter energy function so that there is no need to search for the weighting factors imposed on the original energy function. As a result, the proposed algorithm appears to converge rapidly to the desired solution. Moreover, the designed CFHNN neural-network-based approach is a self-organized structure that is highly interconnected and can be implemented in a parallel manner. It can also be designed for hardware devices to achieve very high-speed implementation.

\section{APPENDIX}

It is always true that a stable state is to be converged in CFHNN evolutions. So, proof of the convergence of the CFHNN is described as follows. The scatter energy function is first considered.

$$
\begin{aligned}
E= & \frac{1}{2} \sum_{x=1}^{n} \sum_{i=1}^{c}\left(\mu_{x, i}\right)^{m}\left|\mathbf{z}_{x}-\sum_{y=1}^{n} \frac{1}{\sum_{h=1}^{n}\left(\mu_{h, i}\right)^{m}} \mathbf{z}_{y}\left(\mu_{y, i}\right)^{m}\right|^{2} \\
& +\frac{1}{2} \nu \sum_{x=1}^{n} \sum_{i=1}^{c}\left(\mu_{x, i}\right)^{m} \tanh \left(\alpha_{i}\right)
\end{aligned}
$$

since $0 \leq\left(\mu_{x, i}\right)^{m} \leq 1$ and $0 \leq \tanh \left(\alpha_{i}\right) \leq 1$; Eq. (17) which implies that

$$
E \leq \frac{1}{2} \sum_{i=1}^{c} \sum_{x=1}^{n}\left[\mathbf{z}_{x}-\sum_{y=1}^{n} \frac{1}{\sum_{h=1}^{n}\left(\mu_{h, i}\right)^{m}} \mathbf{z}_{y}\left(\mu_{y, i}\right)^{m}\right]^{2}+\frac{1}{2} \nu \cdot n \cdot c .
$$


Equation (18) shows that the objective energy is less than or equal to the half of the total distance between training patterns to the cluster centers plus a constant $\frac{1}{2} \nu \cdot n \cdot c$. This proves that $E$ is bounded from below.

Equation (17), same as Eq. (6), is based on least-squared errors criteria, and it is rewritten as follows:

$$
E=\frac{1}{2} \sum_{i=1}^{c} \sum_{x=1}^{n}\left(\mu_{x, i}\right)^{m}\left|\mathbf{z}_{x}-\mathbf{w}_{i}\right|^{2}+\frac{1}{2} \nu \sum_{x=1}^{n} \sum_{i=1}^{c}\left(\mu_{x, i}\right)^{m} \tanh \left(\alpha_{i}\right)
$$

and

$$
\mathbf{w}_{i}=\sum_{y=1}^{n} \frac{1}{\sum_{h=1}^{n}\left(\mu_{h, i}\right)^{m}} \mathbf{z}_{y}\left(\mu_{y, i}\right)^{m}
$$

where $\mathbf{w}_{i}$ (center of cluster $i$ ) is the total interconnection weight received from all neurons $y$ in the same column $i$. As proved in Ref. 25 and described previously, the energy of CFHNN equals $J_{\mathrm{CFCM}}$ as follows

$$
E=J_{\mathrm{CFCM}} \text {. }
$$

Thus the reassignment of a membership degree belonging to cluster $i$ in training vector $\mathbf{z}_{x}$ will result in a decrease of the objective energy function whenever $\mathbf{z}_{x}$ is located closer to a feasible cluster center. In addition, the compensated term will speed up the decrease of the objective function. Consequently, the CFHNN will converge to a satisfactory result after several iterations of updating the reassignment matrix.

\section{ACKNOWLEDGMENT}

This work was in part supported by the National Science Council, ROC, under the Grant\#NSC87-2213-E-167-004, and in part by the image compression research group, National Chin-Yi Institute of Technology, Taichung, Taiwan, R.O.C.

\section{REFERENCES}

1. S. C. Amatur, D. Piriano and Y. Takefuji, "Optimization neural networks for the segmentation of magnetic resonance images," IEEE Trans. Med. Imag. 11 (1992) $215-220$.

2. L. L. H. Andrew and M. Palaniswami, "A unified approach to selecting optimal step lengths for adaptive vector quantizers," IEEE Trans. Commun. 44 (1996) 434-439.

3. J. C. Bezdek, Fuzzy Mathematics in Pattern Classification, Ph.D. Dissertation, Applied Mathematics, Cornell University, Ithaca, NY (1973).

4. C. Y. Chang, R. Kwok and J. C. Curlander, "Spatial compression of seasat SAR images," IEEE Trans. Geosci. Remote Sens. 26 (1988) 673-685.

5. K. S. Cheng, J. S. Lin and C. W. Mao, "The application of competitive Hopfield neural network to medical image segmentation," IEEE Trans. Med. Imag. 15 (1996) $560-567$.

6. P. C. Chung, C. T. Tsai, E. L. Chen and Y. N. Sun, "Polygonal approximation using a competitive Hopfield neural network," Patt. Recogn. 27 (1994) 1505-1512.

7. J. C. Dunn, "A fuzzy relative of the ISODATA process and its use in detecting compact well-separated clusters," J. Cybern. 3 (1974) 32-57. 
8. P. Floreen and P. Orponen, "Attraction radii in binary Hopfield nets are hard to compute," Neural Comput. 5 (1993) 812-821.

9. A. Gersho and R. M. Gray, Vector Quantization and Signal Compression, Kluwer Academic Publishers, Norwell, MA, 1992.

10. R. M. Gray, "Vector quantization," IEEE ASSP Mag. 1 (1984) 4-29.

11. J. J. Hopfield, "Neural networks and physical systems with emergent collective computational abilities," Proc. Nat. Acad. Sci. USA 79 (1982) 2554-2558.

12. J. J. Hopfield and D. W. Tank, "Neural computation of decisions in optimization problems," Biol. Cybern. 52 (1985) 141-152.

13. C. C. Jou, "Fuzzy clustering using fuzzy competitive learning networks," IEEE Int. Conf. on Neural Networks, Vol. II, 1992 pp. 714-719.

14. N. B. Karayiannis and P.-I. Pai, "Fuzzy vector quantization algorithms and their application in image compression," IEEE Trans. Imag. Process. 4 (1995) 1193-1201.

15. J. S. Lin, K. S. Cheng and C. W. Mao, "Multispectral magnetic resonance images segmentation using fuzzy Hopfield neural network," J. Biomed. Comput. 42 (1996) 205-214.

16. J. S. Lin, K. S. Cheng and C. W. Mao, "A fuzzy Hopfield neural network for medical image segmentation," IEEE Trans. Nucl. Sci. 43 (1996) 2389-2398.

17. Y. Linde, A. Buzo and R. M. Gray, "An algorithm for vector quantizer design," IEEE Trans. Commun. COM-28 (1980) 85-94.

18. A. N. Netravali, Digital Pictures: Representation and Compression, Plenum Press, NY, 1988.

19. E. A. Riskin, T. Lookabaugh, P. A. Chou and R. M. Gray, "Variable rate vector quantization for medical image compression," IEEE Trans. Med. Imag. 9 (1990) 290-298.

20. E. Ruspini, "Numerical methods for fuzzy clustering," Inf. Sci. 2 (1970) 319-350.

21. J. E. Steck and S. N. Balakrishnan, "Use of Hopfield neural networks in optimal guidance," IEEE Trans. Aerosp. Electron. Syst. 30 (1994) 287-293.

22. C. T. Tsai, Y. N. Sun, P. C. Chung and J. S. Lee, "Endocardial boundary detection using a neural network," Patt. Recogn. 26 (1993) 1057-1068.

23. T. Washizawa, "Application of Hopfield network to saccades," IEEE Trans. Neural Net. 4 (1993) 995-997.

24. E. Yair, K. Zeger and A. Gersho, "Competitive learning and soft competition for vector quantizer design," IEEE Trans. Sign. Process. 40 (1992) 294-309.

25. M. S. Yang, "On a class of fuzzy classification maximum likelihood procedures," Fuzzy Sets Syst. 57 (1993) 365-375.

26. M. S. Yang and C. F. Su, "On parameter estimation for normal mixtures based on fuzzy clustering algorithms," Fuzzy Sets Syst. 68 (1994) 13-28.

27. L. A. Zadeh, "Fuzzy sets," Inform. Contr. 8 (1965) 338-353.

28. K. Zeger, J. Vaisey and A. Gersho, "Globally optimal vector quantizer design by stochastic relaxation," IEEE Trans. Sign. Process. 40 (1992) 310-322. 


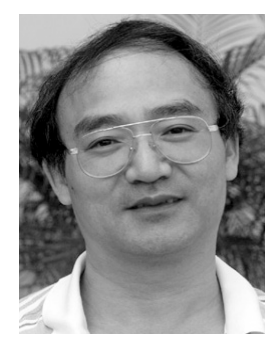

Shao-Han Liu is a Lecturer of the Department of Electronic Engineering at National Chin-Yi Institute of Technology, Taichung, Taiwan, R.O.C. He received the B.S. degree in electronic engineering from Taiwan University of Science and Technology in 1983, and the M.S. degree in Management Science from Northrop University, USA, in 1989.

His research interests involve neural network and image compression.

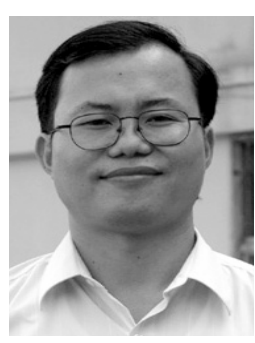

Jzau-Sheng Lin is an Associate Professor of the Department of Electronic Engineering at National ChinYi Institute of Technology, Taichung, Taiwan, R.O.C. He received the B.S. degree in electronic engineering from Taiwan University of Science and Technology in 1980, the M.S. and Ph.D degrees in electrical engineering from National Cheng Kung University in 1989 and 1996 respectively.

His research interests involve neural networks, image compression, pattern recognition and medical image analysis. 